Kairos. Journal of Philosophy \& Science 21, 2019

Center for the Philosophy of Sciences of Lisbon University

\title{
Permissão Para Degradar: Uma Análise Crítica Dos Biodiversity Offsets Nas Práticas Conservacionistas
}

\author{
Rodrigo Muniz \\ Climate Change Impacts, Adaptation and \\ Modelling (CCIAM), Centre for Ecology, Evolution \\ and Environmental Changes (cE3c), Faculdade \\ de Ciências, Universidade de Lisboa (FCUL) \\ \& Instituto Federal de Educação, Ciência e \\ Tecnologia do Sudeste de Minas Gerais - \\ Campus Barbacena (IF Sudeste MG) \\ rmusilva@fc.ul.pt
}

Resumo A conservação da biodiversidade tornou-se um compromisso inquestionável diante do seu papel inexorável para a manutenção da vida na Terra. Apesar do consensual compromisso em sustentá-la, as razões pelas quais fazê-lo ainda são alvo de discussões e controvérsias. Que valores devem reger sua proteção? Contudo, a conservação da biodiversidade está cada vez mais voltada para um lado objetivo-econômico, na qual a natureza é marginal aos interesses que parecem mais evidentes: a eficiência econômica. Esse artigo faz uma reflexão sobre os esquemas de biodiversity offsets, instrumentos cujo objetivo é a compensação pelos danos à biodiversidade provocados pelas atividades de desenvolvimento; e sobre a mudança nos valores da conservação da biodiversidade que tais instrumentos tendem a provocar. Uma mudança discursiva, institucional, técnica e material que altera o sentido de se fazer conservação e a relação humana com o mundo natural. Essa 'nova' perspectiva da conservação revela dilemas éticos fundamentais, e vem se tornando uma estratégia cada vez mais difundida no âmbito das políticas ambientais para a conservação.

Abstract The biodiversity conservation became an unquestionable commitment facing its inexorable role to maintain the life on Earth. Despite the consensual commitment to sustain biodiversity, the reasons why to do it are still subject of discussions and controversies. What values should lead it protection? However, the biodiversity conservation is increasingly turned to an economic-objective bias, in which nature is marginal to the interests that seem most obvious: economic efficiency. This arti- 
cle reflects on biodiversity offsets schemes, instruments aimed to compensate the damages to biodiversity caused by development activities; and also reflects on the changes in the values of biodiversity conservation, that such instruments tend to cause. It is a discursive, institutional, technical and material change, shifting the meaning of conservation and the human relationship with the natural world. This 'new' conservation perspective reveals fundamental ethical dilemmas and is becoming an increasingly widespread strategy in the context of environmental policies for conservation.

DOI 10.2478/kjps-2019-0003

\section{Introdução: Valores na Conservação da Natureza e da Biodiversidade}

Para se entender a história da conservação, requer-se antes defini-la pelos seus objetivos, não pelos seus efeitos.' Os seres humanos sempre exerceram impacto sobre o mundo natural não-humano, mas nem sempre foram considerados conservacionistas. Um grupo que vive em relativo equilíbrio com seu meio natural não Ihe causa danos, uma vez que este produz mais do que o grupo precisa. É igualmente nesse sentido que o historiador ambiental Warren Dean aponta que os povos indígenas não eram conservacionistas, em termos de proteção das florestas para a geração vindoura. ${ }^{2}$ Não era uma relação de descuido, nem desrespeito, mas havia uma certeza de adequação das fontes naturais às suas necessidades e de sua capacidade de defendê-los dos seus competidores. A floresta que havia bastava para sua boa vida.

Diferentemente, hoje, deparamo-nos com a sexta extinção em massa, causada por nós seres humanos. ${ }^{3}$ Esta é mais uma evidência, daquilo que se tem entendido por Antropoceno, nova época geológica, em que o ser humano se tornou uma força motriz geofísica. ${ }^{4}$ Começa-se a se perceber uma interiorização antrópica: o mundo natural passa a fazer parte do mundo humano, e não mais o mundo humano parte do mundo natural. ${ }^{5}$ Considerando a capacidade humana de dizimar tamanha diver-

\footnotetext{
$1 \quad$ Van Dyke, 2008.

2 Dean, 1996.

3 Pievani, 2014.

4 Crutzem e Stoermer, 2000.

5 Viveiros de Castro, 2013.
} 
sidade biológica, o biólogo conservacionista Michel Soulé sugeriu ironicamente, ao invés de Antropoceno, outra designação: "Catastrophozoic", a era 'Catastrofozóica'. ${ }^{6}$ Como lidar com as mudanças globais pelas quais atravessamos, que na maioria das vezes infligimos?

Para a conservação, um dos aspetos fundamentais é lidar com a perda da biodiversidade, sobretudo em um contexto de alterações globais, cada vez mais intensificadas pelas ações humanas. ${ }^{7}$ Preservar a biodiversidade é um compromisso que assume uma importância inquestionável, pois representa um papel inexorável para a manutenção da vida na Terra, incluindo a humana. Historicamente, não houve motivações conservacionistas, por mais que as relações entre seres humanos pudessem em alguns casos representar uma relação de respeito, nunca houvera, portanto, necessidade de conservar. A conservação é uma tarefa moderna ${ }^{8}$, e apenas quando os seres humanos começam a reconhecer na natureza um valor por ela mesma é que surge a ideia de conservação, descreve Van Dyke (2008). ${ }^{9}$ A biologia da conservação surge como uma ciência que se estabeleceu enquanto uma resposta à perda da biodiversidade que rapidamente se alastrava no século passado. ${ }^{10} \mathrm{Um}$ de seus postulados é de que a diversidade biótica possui valor intrínseco, que independe de seu valor instrumental ou utilitário, nas palavras de Soulé (1985, p. 731): "Species have value in themselves, a value neither conferred nor revocable, but springing from a species' long evolutionary heritage and potential or even from the mere fact of its existence". "O'Neill (1993; 2005) realça que o termo 'intrínseco' poderá expressar-se em variados

$6 \quad$ Soulé, 1996.

7 Quando falamos em conservação da biodiversidade, também corremos o risco de ser reducionistas. A biodiversidade é um valor natural, mas não é o único. Assim, não se deve proteger apenas a diversidade biológica (por exemplo, a riqueza e diversidade de espécies), mas todo o sistema de valores naturais, isto é, todo sistema que permite que a vida floresça em suas diversas manifestações. Contudo essa questão exigiria mais tempo do que dispomos. Portanto, de modo a tornar mais simples o debate sobre os valores na conservação, o termo biodiversidade, aqui, representa o conjunto de valores naturais de uma forma geral, a não ser que seja especificado de outra forma ao longo do texto.

$8 \quad$ Larrère e Larrère, 1997.

9 Van Dyke, 2008.

10 Soulé, 1985; Wilson, 2002.

11 Soulé, 1985, 731. 
sentidos. Aqui, o valor 'intrínseco' significa um valor independente de uma referência instrumental, isto é, independente de qualquer utilidade que possa representar para os seres humanos. ${ }^{12}$

Há, no entanto, tamanha diversidade de valores em torno da natureza que falar apenas do valor intrínseco que ela representa poderá parecer redutor, especialmente ao considerarmos as subjetividades que revestem a capacidade humana de valorar. Assim, a natureza poderá despertar uma diversidade de valores, conforme nossa cultura também se organiza, tais como o valor de suporte de vida, valor recreativo, valor científico e educacional, valor estético, valor sagrado, terapêutico, histórico e identitário, económico, entre outros. ${ }^{13}$ Ao admitir tal pluralidade de valores, inevitavelmente admite-se imensa complexidade em suas relações. ${ }^{14} \mathrm{O}$ que o ser humano valora e no nível em que valora depende dos próprios valores de quem valora e do valor das coisas em si próprias. Os valores variam consoante os desejos e inclinações e isso reflete os valores incorporados na cultura humana. ${ }^{15}$ Mas quanto mais valores e interesses humanos associados à causa da conservação parece igualmente crescer a divergência com o dever de se preservar a biodiversidade. Essa tensão ainda segue bastante acesa, contudo com novas nuances e dimensões. ${ }^{16}$ Portanto, é verdade que há tensões nos valores que circundam e suportam a conservação, não obstante, os valores são centrais no tema da conservação. ${ }^{17}$

Nesse sentido, apesar do consensual compromisso em sustentar a biodiversidade, as razões pelas quais fazê-lo ainda são alvo de discussões

\footnotetext{
12 O’Neill 1993; O’Neill 2005.

13 Rolston, 1989; Holland e Rawles, 1994.

14 A teoria do valor em um sentido mais amplo atravessa variados âmbitos (filosofia moral, social, política estética, feminista, da religião, entre outros), nos quais se possa encontrar algo sujeito à valoração. Em um sentido mais estreito, a teoria do valor corresponde, sobretudo, às temáticas da Axiologia. Cf. Schroeder, Mark, "Value Theory", The Stanford Encyclopedia of Philosophy (Summer 2012 Edition), Edward N. Zalta (ed.), <http://plato.stanford.edu/archives/sum2012/entries/value-theory/>. Acessado Dezembro 2018.
}

15 Foster, 1997.

16 Minteer e Miller, 2011 ; Miller et al., 2011.

17 Sandbrook et al., 2011. 
e controvérsias. ${ }^{18} \mathrm{O}$ desacordo sobre as motivações de proteção espelha-se, sobretudo, nos valores que são atribuídos e reconhecidos à biodiversidade. Que valores devem reger sua proteção? ${ }^{19} \mathrm{Um}$ valor instrumental (valores que existem apenas a partir do olhar humano) ou um valor intrínseco (um valor independente do olhar humano)? Este é ainda um debate central na ética ambiental. A ética na conservação, isto é, como nós seres humanos devemos nos relacionar com o mundo natural, é relativamente recente, tal como a propria ideia de conservação.

O comprometimento ético destacou-se na conservação, na medida em que se lançava a expansão da considerabilidade moral da vida na terra, sobretudo no final da década de 1960 e início de 1970. As perspetivas éticas ambientais não antropocentradas ${ }^{20}$ passaram a influenciar com outro teor o pensamento da conservação, relevando, sobretudo, as razões intrínsecas para a conservação da natureza. Naturalmente, o direito humano a um ambiente íntegro é, por certo, um dos principais objetivos da biologia da conservação. Assim, a ética da conservação, sob o olhar humano, dirá que os ecossistemas também possuem um valor em nome de sua contribuição para as experiências humanas (isto é, um valor antropocêntrico). Os seres humanos têm, certamente, o direito de florescer na terra, mas nos será dado o direito de degradar, ou devastar por completo os ecossistemas? ${ }^{21}$

Não obstante, o debate da literatura acerca dos valores ambientais ${ }^{22}$, dentre os quais se enquadra a ética para a conservação, ainda insiste na discussão sobre a existência, ou não, de valores em torno da natureza ${ }^{23}$,

\footnotetext{
18 Norton, 2000.

19 Rosa e Marques da Silva, 2005.

20 Beckert e Varandas, 2004.

21 Rolston III, 1995.

22 O'Neill et al. 2008. Curiosamente os autores apontam que, de certa forma, não há algo que se possa chamar de valor. Ao invés, há variadas formas nas quais os indivíduos, processos e lugares importam a nós, assim como as variadas formas de nos relacionarmos com estes, e as variadas considerações que integram nossas deliberações aquando das ações. Portanto, argumentam que os ambientes, no plural, e suas constituintes, bem ou mal, importam para nós de formas distintas. É interessantes realçar que a corrente econômica prevalecente apropriou-se do termo valor, atendendo às suas especificidades, relacionando-o com um valor transacionável, praticamente opondo-se à sua conceituação mais abrangente, que engloba os valores éticos, estéticos e espirituais, por exemplo.
}

23 Holland e Rawles, 1996. 
se tais valores são objetivos ou subjetivos à natureza. Nesse sentido, a busca pela conservação pode ser uma busca humana na qual se incluem considerações culturais, tradicionais e sobre a própria subsistência. Em última instância, também o valor intrínseco que se reconhece no mundo natural pode representar o desejo humano de conservar. Ainda que o que lá está possua um valor intrínseco, existe uma comoção naquele que contempla. A habilidade humana de valorar, fonte desta comoção do contemplador, é um valor antropogénico, pois implica subjetividade, mesmo que este valor não seja antropocêntrico. ${ }^{24}$

Se a natureza humana implica a pluralidade de valores, também implica serem plurais as deliberações, decisões e ações humanas. É certo que o ser humano age de maneiras diversas e são variados os critérios éticos e morais que o podem guiar. Em certas situações age por uma lógica utilitarista (consequencialista), em determinados momentos guia-se pela lógica deontológica (do dever) e, por vezes, age pela virtude (inclinação do caráter para o bem). Também a ética ambiental influencia o agir humano, ampliando a comunidade ética, de maneira a contemplar outras entidades não-humanas, como a natureza em si mesma. É importante reconhecer o equilíbrio, ao menos em um nível reflexivo, entre essas correntes éticas e seus desdobramentos na cadeia relacional humana, de modo a buscar maior clareza ética. Os valores se relacionam com os interesses dos agentes, considerando que a natureza contribui para nossa sobrevivência e prosperidade, mas não apenas centrados na natureza enquanto recurso, considerando também sua beleza e transcendência, mas também nos é possível igualmente destacar a importância da natureza livre da influência dos interesses humanos.

No entanto, as discussões sobre a valoração da natureza que mais tem ganhado energia nos últimos anos, fundamenta-se, principalmente, na oposição entre a teoria do valor intrínseco e a teoria económica que resume os valores naturais aos valores económicos, que tornam secundários não apenas o valor intrínseco, mas outros valores socioculturais fundamentais nas relações humanas com o mundo natural não-humano.

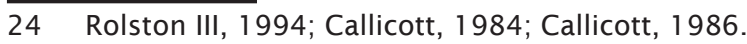


Esse artigo é um recorte sobre a mudança nos valores da conservação da biodiversidade. É uma mudança discursiva que tem levado a novas formas de perceber e de se relacionar com o mundo natural não-humano em maneira estritamente econômica, sobretudo financeira. É também uma mudança institucional que induz os arranjos institucionais (redes e alianças entre o mundo corporativo, financeiro e conservacionista) a se acomodarem na tarefa de tornar reais as metáforas do 'capital natural' e dos 'serviços ecossistémicos'. ${ }^{25}$ É ainda uma mudança técnica e material que visa fragmentar a natureza, assinalar valores monetários aos seus 'fragmentos' e facilitar sua transação através de instrumentos baseados no mercado. A natureza, dessa forma passa a ser transcrita em bens e serviços passíveis de quantificação, transação, degradação e compensação para que, de um modo geral, os danos ambientais possam ser minimizados. Os atuais discursos do 'capital natural' e dos 'serviços dos ecossistemas', linguagens tão popularizadas hoje na conservação, têm possibilitado e representado tais mudanças, apesar de originalmente cunhadas para desempenhar funções pedagógicas e metafóricas. ${ }^{26}$

Aqui se faz, sobretudo, uma reflexão acerca dos biodiversity offsets, família de instrumentos e mecanismos de conservação baseados em mercado, que são implementados para compensar os danos residuais à biodiversidade provocados por um empreendimento. Essa perspetiva economicista neoliberal da conservação ${ }^{27}$ revela importantes dificuldades éticas, técnicas, sociais e de governança ${ }^{28}$ (Maron et al., 2016), alterando a perceção humana acerca do mundo natural ${ }^{29}$ e, muito rapidamente, tem se tornado uma abordagem política ambiental relevante no âmbito da conservação.

25 Sullivan, 2013; Sullivan, 2014.

26 Schumacher, 1973; Gómez-Baggethun et al., 2010; Noorgard, 2010.

27 Spash, 2009; Büscher et al., 2012; Spash, 2015.

28 Maron et al., 2016.

29 Sullivan, 2010; Hannis e Sullivan, 2012; Spash, 2015. 


\section{Biodiversity Offsets: Permissão Para Degradar Nas Novas Práticas Conservacionistas?}

A conservação atravessa uma fase de reforma que acompanha as tendências do crescimento económico, uma fase na qual "[...] há sempre uma presunção a favor do desenvolvimento". ${ }^{30} \mathrm{O}$ planeamento do uso da terra, e consequentemente a conservação, deve, segundo essa presunção, encorajar o crescimento económico, ao invés de restringi-lo. ${ }^{31}$ É na esteira desse pressuposto que os novos instrumentos baseados em mercado para a conservação passam a despertar maiores interesses e a ganhar visibilidade, como é o caso dos mecanismos e instrumentos designados biodiversity offsets, ou as chamadas compensações pelos danos causados à biodiversidade. A ideia é estimular o estabelecimento de um empreendimento, mesmo quando este represente um perigo para a biodiversidade, na medida em que compensar este dano se torna possível.

O que são afinal os instrumentos e macanismos denominados biodiversity offsets? Uma das definições mais citadas ao longo da literatura é a da organização Business and Biodiversity Offsets Programme $e^{32}$ que os caracteriza como:

\section{...measurable conservation outcomes resulting from} actions designed to compensate for significant residual adverse biodiversity impacts arising from project development after appropriate prevention and mitigation measures have been taken. The goal of biodiversity offsets is to achieve no net loss and preferably a net gain of biodiversity on the ground with respect to species composition, habitat structure, ecosystem function and people's use and cultural values associated with biodiversity.

O termo offset é frequentemente utilizado para denominar uma série de instrumentos, mecanismos e políticas, tais como a criação compen-

30 Hannis e Sullivan (2012) afirmam que essa presunção é uma retórica reminiscência da era Thatcher. Trata-se de uma passagem do White Paper 'Lifting the Burden' da gestão Margareth Thatcher, disponível em: http://legacy.library.ucsf.edu/tid/noz86a99/pdf.

31 Hannis e Sullivan, 2012.

32 Business and Biodiversity Offsets Programme, 2009. 
satória de habitat (compensatory habitat creation $^{33}$ ), os bancos de mitigação (mitigations banks ${ }^{34}$ ), os bancos de conservação, os bancos de habitat, os bancos de biodiversidade (conservation banking, habitat banking, biodiversity banking ${ }^{35}$ ), entre outros. Offset banks são os 〈lugares〉 nos quais são criados os projetos de compensações para se trocar por créditos de biodiversidade e consequentemente compensar os danos ecológicos gerados por um projeto de desenvolvimento. ${ }^{36}$

Os esquemas de biodiversity offsets (BDO, daqui por diante) são muito semelhantes às compensações carbônicas para a mitigação ou redução dos impactos tardios das alterações climáticas. ${ }^{37}$ Vale ressaltar que os gases com efeito de estufa são avaliados de forma relativamente uniforme, facilitando sua transação enquanto commodity no nível internacional $^{38}$, e ainda assim estão repletos de complexidades, singularidades e dificuldades ${ }^{39}$, o que dizer sobre as transações de biodiversidade propostas nas operações dos esquemas de BDO?

Essas medidas tendem a abstrair a complexidade da biodiversidade a indicadores representativos, como um habitat ou uma espécie, de modo que possam ser compensados em qualquer outro sítio. Portanto, os mecanismos de BDO ocorrem, sobretudo, a partir de tradeoffs entre elementos da biodiversidade ${ }^{40}$, nos quais uns até podem ser beneficiados em relação a outros (por exemplo, endemismo, insubstituibilidade ou qualquer outra característica que revele vulnerabilidade ou que dê um sentido de singularidade). Contudo, é mais frequente que o proxy seja vislumbrado através de uma métrica única, como o valor monetário para quantificar a biodiversidade. ${ }^{41} \mathrm{O}$ conceito de BDO, assim como qualquer outro instrumento baseado em mercado, implica delinear fronteiras físi-

\footnotetext{
33 Morris et al., 2006.

34 Gibbons e Lindenmayer, 2007.

35 Madsen et al., 2011.

36 Bull et al., 2013.

37 Waeber, 2013.

38 ten Kate et al., 2004.

39 Böhm e Dabhi, 2009.

40 Castree, 2003; Waeber, 2013.

41 Waeber, 2013.
} 
cas para as funções e os 'serviços dos ecossistemas' e assinalar um preço virtual aos elementos sistémicos ou aos valores (intrínseco ou cultural, por exemplo) para relativizar sua contribuição para a biodiversidade como um todo. ${ }^{42}$

Curiosamente, uma das premissas para a conceptualização da biodiversidade enquanto crédito é a impossibilidade de se definir uma unidade suficientemente consistente para entender a biodiversidade. ${ }^{43}$ É nesse sentido que a biodiversidade é compreendida através de proxys, como um habitat, por exemplo. Assim, os 'bancos de habitat' facilitam a transação de habitats, pois operam tal como um sistema bancário, não obstante para facilitar as negociações de créditos de biodiversidade. Apesar disso, os créditos de habitat são adquiridos através do valor monetário, por meio da financeirização da biodiversidade. Aqueles que trabalham mais pragmaticamente com o valor estabelecido na economia de mercado entendem que o valor monetário, dentro de uma estrutura numérica, é a forma mais eficiente de dar visibilidade para a natureza. Enquanto outros entendem que essa mudança de perspetiva sobre a natureza não reflete apropriadamente a singularidade e a consequente insubstituibilidade de espécies, populações e habitats, como confere o valor intrínseco e outros valores socioculturais adjacentes. ${ }^{44}$ Como descrevem O'Neill et al. (2008), o dinheiro provê a unidade e a análise de custo e benefício é o método utilizado para comparar os valores e assistir a tomada de decisão. É uma abordagem que, pela regra utilitarista, acaba por substituir as complexidades que existem entre os diversos valores e objetivos, que competem entre si, por análise homogênea. A unidade monetária não é capaz de representar uma pluralidade de valores, oferecendo-se mais como uma medida para troca do que uma medida para comparar diferentes valores. ${ }^{45}$

Portanto, não é uma tarefa fácil medir as perdas e ganhos para a biodiversidade. Assim, as formas para se medir esses aspetos variam de um para outro, mas podem incluir funções ecológicas, tamanho e vari-

42 Waeber, 2013; Kosoy e Corbera, 2010.

43 Bull et al., 2013.

44 Hannis e Sullivan 2012; McCauley, 2006; Spash, 2015.

45 O'Neill et al., 2008. 
abilidade de espécies ameaçadas, bem como a qualidade das vegetações e tipos de habitats associados. ${ }^{46}$ No entanto, costuma ser relevante, ao menos teoricamente, aquilo que se denomina equivalência ecológica, isto é, o ganho deve corresponder ao tipo de biodiversidade que sofreu o dano. ${ }^{47}$

Organizações de todo o globo (incluindo organizações conservacionistas) têm proposto e utilizado cada vez mais instrumentos baseados na compensação da perda da biodiversidade em diversas escalas, incluindo a escala supranacional, permitindo e difundindo a conservação através da criação de bancos para a biodiversidade. ${ }^{48}$ Do mesmo modo, a acompanhar a difusão dessas práticas, cresceu também o número de publicações. ${ }^{49}$

Sendo assim, é essencial lançar um olhar crítico sobre esses instrumentos, na medida em que ao longo da constituição da metáfora e de sua transição para a realidade, as abstrações economicistas da natureza (tais como as metáforas do capital natural e dos serviços dos ecossistemas, apropriados pela linguagem economicista) são entendidas como designações isentas de reconsideração, sendo essa uma das razões pelas quais a conservação passa a ser praticada sob este viés. ${ }^{50}$ Nessa medida, o conceito cria um padrão de ordens de pensamento entendido como

46 Maron et al., 2012.

47 Também chamado de 'in kind' ou 'like-for-like' offsets, Maron et al., 2012.

48 Lapeyre et al., 2015; Madsen et al., 2011. Algumas iniciativas já em prática: Conservation (Species) Banking and Wetland and Stream Mitigation nos Estados Unidos da América; programa HADD - Harmful alteration, disruption or destruction, de compensação de habitats de peixes no Canadá; compensação de ativos ambientais de Reservas Legais do Código Florestal Brasileiro e entre empreendedores; Política Nacional de Compensação da África do Sul; Projetos da BBOP em Madagascar; Regulação da Mitigação de Impactos (Eingriffregelung) na Alemanha, CDC (Caisse des dépôts et consignations ) Banco de Biodiversidade na França e outras regiões da Europa; o Upland Mitigation Bank em Saipan (EUA); o mercado voluntário Malua BioBank na Ásia; os programas South Wales BioBanking e Queensland's Koala Offsets na Austrália; dentre muitos outros.

49 Maron et al., 2015.

50 O pensamento economicista envolve o pensar de maneira estritamente econômica, centrado exclusivamente nas questões de produção e consumo, no qual é fundamental o cálculo econômico e, no que concerne à natureza, deve ser tratada como um bem para ser considerado neste cálculo. O pensamento economicista também pode tentar considerar outros elementos (não econômicos). Contudo, para tal não reconhece um limite para o seu delineamento, trazendo a natureza para consideração sob uma abordagem econômica. Por exemplo, os elementos naturais passam a ser abordados enquanto capital 
uma realidade incontestada, nos quais os aspetos socialmente fabricados são ocluídos. ${ }^{51}$ Esse mito ${ }^{52}$ parte do pressuposto de que a integração da economia com a ecologia (ou com a conservação, aqui no caso) é condicionada por uma fragmentação conceptual dos constituintes da natureza que implica separá-los do seu contexto ecológico, de suas relações sócio-ecológicas e de diferentes conceções de valor. ${ }^{53} \mathrm{E}$ mais do que um status de commodity para o mundo natural ${ }^{54}$, a criação e a materialização do capital natural suporta uma financialização da natureza não-humana através de sua inscrição discursiva, técnica, institucional e material enquanto dinheiro. ${ }^{55} \mathrm{O}$ mito económico da natureza vista como capital natural e provedora de serviços se torna rapidamente uma nova ordem hegemônica de perceção do mundo natural. Sendo assim, a conservação se volta não para a natureza, ou mesmo para a biodiversidade, mas para outras entidades: o capital e os serviços. Essa mudança conceptual não é inócua, possui implicações práticas no desenho das políticas de conservação e nos instrumentos e mecanismos que se passam a utilizar tendencialmente.

\section{Princípios Em Causa: Uma Crítica Aos ‘Princípios’ Da Compensação}

Maron et al. (2016) realçam que os BDO apresentam dificuldades éticas, sociais, técnicas e de governança que precisam ser endereçadas de modo a minimizar os riscos que tais mecanismos apresentam para a conservação. O primeiro desafio, incontornável, que os BDO levantam são os valores que os constituem, de caráter ético/filosófico. Parte das discussões ético/filosóficas no âmbito dos biodiversity offsets pode distinguir essencialmente dois posicionamentos: (i) a defesa do valor intrínseco da natureza; e (ii) a defesa dos valores da natureza para os seres

e serviços para serem considerados no âmbito econômico (Schumacher, 1973; Foster, 1997).

51 Sullivan, 2014.

52 Midgley, 2004.

53 Sullivan, 2014.

54 Castree, 2003; Kosoy e Corbera, 2012.

55 Sullivan, 2013; Sullivan, 2014. 
humanos (isto é, um valor instrumental). ${ }^{56} \mathrm{~A}$ simplificação e redução da natureza em unidades transacionáveis é uma violação de seu valor intrínseco. ${ }^{57}$ Esta é um das principais objeções da defesa do valor intrínseco. Deste modo, não poderá haver um resultado neutro de uma compensação da biodiversidade, uma vez que qualquer dano causado (quer seja ao nível do individuo, da espécie ou do ecossistema) afeta entidades únicas, trazendo implicações morais. ${ }^{58}$

Como já referido, os BDO são considerados instrumentos que revelam a expansão do mercado e seus valores sobre a natureza e sua conservação. ${ }^{59} \mathrm{~A}$ transição da abordagem regulatória para as os instrumentos baseados em mercado que flexibilizam a perda da biodiversidade representa uma mudança significativa nos valores da conservação. ${ }^{60}$ Argumenta-se que essa lógica promove uma permissão para degradar, pois demove a ética que contesta a destruição da natureza, e potencialmente leva à aceitabilidade social da destruição da natureza e da biodiversidade. ${ }^{61}$

São muitos os princípios que correspondem à constituição de uma plataforma conceptual para a transação da biodiversidade e, portanto, da natureza. Hannis e Sullivan (2012) analisam cinco princípios fundamentais ${ }^{62}$ para demonstrar os impactos dos BDO à conservação, desde as dificuldades éticas às técnicas. Essa secção está organizada em torno destes princípios.

O primeiro desses princípios é a 'hierarquia de mitigação e a lógica circular da inevitabilidade'. Partindo da definição da Business and Biodiversity Offsets Programme (BBOP) ${ }^{63}$, uma das principais instituições

\footnotetext{
56 Maron et al., 2016.

57 Rolston, 1994; Spash, 2009; Maron et al., 2016.

58 Rolson, 1994; Maron et al., 2016.

59 Büscher et al., 2012; Spash, 2015.

60 Maron et al., 2016.

61 Moreno-Mateos et al., 2015; Ives e Bekessy, 2015; Maron et al., 2016.

62 i) the mitigation hierarchy and the circular logic of 'unavoidability'; ii) the legitimation of off-site mitigation; iii) the principle of additionality; iv) the construction of metrics that create apparent commensurability between places; v) the key role of enabling policy and governance frameworks.
}

63 BBOP, 2009; BBOP, 2012. 
globais promovedoras dos BDO, Bull et al. (2013) afirmam que BDO são ações que visam criar ganhos adicionais comparáveis de biodiversidade de modo a compensar a perda causada pelos projetos de desenvolvimento. Essas medidas procuram compensar uma perda inevitável, na sequência de uma hierarquia de mitigações. Por isso uma medida de BDO pode requerer, antes, 'evitar, minimizar e reabilitar (restaurar)', qualquer impacto sobre a biodiversidade o máximo possível, para que as compensações possam se aplicar aos impactos residuais. Portanto, fica entendido que os esquemas de BDO tomam lugar no final de uma hierarquia de medidas relativamente às atividades que geram danos ao ambiente natural, nomeadamente: i) evitar; ii) minimizar; iii) mitigar (restauração e compensação). Os esquemas de BDO se enquadrariam na categoria de mitigação, sobretudo na compensação. ${ }^{64}$

É este último recurso da hierarquia dos BDO que permite que seja estimulada a criação de um mercado de créditos para a conservação. Quando o dano de um empreendimento é considerado inevitável, legitima-se a noção de que a biodiversidade pode sempre ser compensada, mitigando-se a degradação à parte do sítio no qual a biodiversidade foi prejudicada, naquilo que se entende por mitigação off-site, ou simplesmente offsetting. É importante também dizer que isso influencia na conceção daquilo que é o 'inevitável', onde e por quê é inevitável. Quem é que decide tais fatores, se é o próprio desenvolvimento a carruagem desses instrumentos? ${ }^{65}$

E ainda que seja reconhecido que a aplicação da hierarquia de mitigação em praticamente todas as recomendações nas políticas de offset, não há regras claras sobre como mover de um estágio para outro na hierarquia. Assim, fica ao encargo dos empreendedores e dos reguladores decidir, caso a caso, com pouca ou nenhuma referência ou orientação se o impacto pode ou não ser evitado ou minimizado, o que igualmente dificulta estimar adequadamente qual será o impacto residual para a aplicação da mitigação. ${ }^{66} \mathrm{E}$ mesmo diante do potencial de uma adequada

64 Maron et al., 2012; Bull et al., 2013; Froger et al., 2015; Lapeyre et al., 2015.

65 Hannis e Sullivan, 2012.

66 Maron et al., 2016. 
compensação a um dano, evitar um dano deverá estar baseado na aceitabilidade social, não em argumentos técnicos e económicos. ${ }^{67}$

O segundo princípio é o da 'mitigação off-site', isto é, uma compensação em lugar distinto do local do empreendimento. Os proponentes dos esquemas de BDO argumentam que ao invés de fragmentar, a mitigação off-site contribui para a conectividade entre os habitats, reforçando o valor e a resiliência ecológicos; e ainda defendem que esta abordagem consegue criar um mosaico mais contínuo, ao contrário das áreas protegidas que se encontram fragmentadas. ${ }^{68}$ Ora, uma das maneiras de se operacionalizar os esquemas de BDO é através da criação de um habitat, obviamente, off-site. Assim, não parece ser apenas uma questão de gerir melhor pequenas parcelas de espaços verdes, mas uma questão de interesses. A oferta e demanda, preços e disponibilidade de créditos serão, muito provavelmente, os principais orientadores da escolha do sítio. Os empreendedores, por exemplo, poderão ser eles próprios a prover compensações ou comissionar outros que o façam por eles. Um fator chave nesses mecanismos de compensação é o fato de que os offsets podem ser trocados, isto é, um empreendedor poderá simplesmente comprar créditos de um proprietário ou de outros gestores de reservas habilitados a acumular créditos através de práticas de restauração, criação e gestão de habitats. ${ }^{69}$

Neste contexto, os habitats representam a biodiversidade, enquanto um proxy. Baseada nesse pressuposto está a ideia do habitat banking. O conceito de "banco de habitat" pode ser definido como uma forma de compensação em que as medidas compensatórias resultantes são transformadas em bens (assets) que podem ser comercializados, criando assim um instrumento de mercado. Isto é, um mercado onde os créditos (de ações com resultados benéficos para a biodiversidade) podem ser comprados para compensar um débito (dano ambiental). Os créditos podem ser produzidos com antecedência (sem ligação necessária com

67 Maron et al., 2016.

68 White, 2008.

69 Hannis e Sullivan, 2012. 
os débitos que vão compensar) e podem ser acumulados ao longo do tempo. O termo pode relacionar-se tanto a espécies como a habitats. ${ }^{70}$

A lógica das compensações é fazer da 'provisão de habitat' um negócio atrativo para os proprietários de terras, na medida em que a demanda pela compensação será estimulada. Portanto, um mercado de offsets poderia incentivar os proprietários de terras e integrar-se proactivamente numa espécie de sistema bancário para a conservação através da criação, restauração ou preservação de remanescentes, esperando um retorno financeiro em uma futura venda de créditos a empreendedores. ${ }^{71}$ São estes créditos (ex-ante ou ex-post) advindos de pequenas parcelas de habitats que supostamente agregariam os benefícios para largas áreas de grande valor ecológico. ${ }^{72}$

É uma conservação guiada pelo desenvolvimento, isto é, acontece sempre em função do desenvolvimento, condicionada aos pressupostos do crescimento econômico. É um mecanismo em que a demanda por créditos está condicionada pela destruição ambiental. Isto é, não há interesses em conservar se, paralelamente, não há danos para se compensar.

O terceiro princípio é o da 'adicionalidade', já utilizada no EU Emissions Trading Scheme direcionado para o carbono e equivalentes, no contexto das alterações climáticas. ${ }^{73}$ Uma atividade que visa a mitigação dos gases com efeito de estufa, por exemplo, deve ter um resultado adicional em relação à ausência dessa atividade. ${ }^{74}$ É um conceito vago, aberto a interpretações variadas e subjetivas. Esta já seria uma das primeiras dificuldades da adicionalidade. A adicionalidade poderia representar, aqui neste contexto, uma redução do dano ambiental em relação àquele estado ambiental no qual não se tomaram tais medidas, os pagamentos, no caso do mercado bancário para a conservação. Ora, os pagamentos

70 Eftec e IEEP, 2010.

71 Um recente relatório do DEFRA (Department for Environmente, Food \& Rural Affairs), do Governo Britânico, relata que biodiversity offsetting é a maior oportunidade no âmbito do negócio ambiental, disponível em: http://webarchive.nationalarchives. gov.uk/20130822084033/http://www.defra.gov.uk/ecosystem-markets/2012/06/27/ vnn-report-published270612/.

72 Hannis e Sullivan, 2012.

73 c.f. http://ec.europa.eu/clima/policies/ets/index_en.htm.

74 United Nations Framework Convention on Climate Change (UNFCCC), 1998. 
são considerados a medida na qual a conservação acontece, gerando a tal adicionalidade. Como pode o pagamento ser o critério que qualifica a conservação? Quem poderá atestar o que teria acontecido sem o pagamento? ${ }^{75}$ Ademais, de acordo com essa definição de adicionalidade, o próprio BDO representaria uma adicionalidade, diante de sua ausência, o que é bastante controverso. ${ }^{76}$

Seguindo ainda a definição da $\mathrm{BBOP}^{77}$, uma característica própria do BDO é a inclusão do conceito de 'no net lost' e o conceito de 'net gain'. ${ }^{78}$ São conceitos que acabam por representar o princípio da adicionalidade. Mais uma importante interpretação que se faz dessa definição é de que as políticas de offset exigem que as compensações sejam 'in kind', ou seja, de um mesmo tipo (uma floresta por uma floresta). Mas isso varia de acordo com o país, regulação e legislação e mesmo interpretação. Dificilmente se poderá compensar um ecossistema complexo como o mangue (ou mesmo um sapal) no mesmo local de um projeto desenvolvimentista industrial, por exemplo. Contudo, todas essas medidas envolvem uma quantificação do impacto ecológico residual derivado de um empreendimento para que seja compensado em um lugar qualquer. ${ }^{79}$ Existem desafios inexoráveis em quantificar os efeitos residuais de um evento que ainda não tomou lugar para se compensar de forma equivalente (especialmente quando off-site).

Para evitar o sobreuso do termo, a BBOP, entre outras organizações envolvidas com os mecanismos de $\mathrm{BDO}^{80}$, considera o conceito de 'no net loss' central para os esquemas de BDO. Mesmo sendo conceitos chave no contexto dos BDO, há grande ceticismo em torno deles. Há argumen-

75 Hannis e Sullivan, 2012.

76 Gillenwater, 2012.

77 BBOP, 2009; BBOP, 2012.

78 'No net lost' significa uma 'perda nula' ou 'sem perda líquida' de biodiversidade em todo o processo de estabelecimento de um projeto de desenvolvimento, enquanto que 'net gain', ainda mais ambiciosamente siginifica um ganho para a biodiversidade, isto é, um impacto positivo após terminado o projeto de desenvolvimento.

79 Bull et al., 2013.

80 BBOP, 2009; BBOP, 2012; The Economics of Ecosystems and Biodiversity (TEEB), 2010; eftec e IEEP, 2010 ; Rio Tinto, 2008. 
tos sob o aspeto ecológico que apontam para a impossibilidade de uma simples métrica poder representar tão precisamente os ecossistemas. ${ }^{81}$

Curiosamente, no esquema de 'species banking' nos Estados Unidos da América, cerca de 107 dos 123 bancos de espécies listados em 2010 preservaram localidades já em estados conservados anteriormente, colocando em cheque o pressuposto da adicionalidade, mas, em contraponto, aumentando o valor financeiro das terras (maioritariamente privadas). ${ }^{82}$ Portanto, não se pode afirmar que aumentaram as áreas de conservação, ou que não houve a chamada 'perda nula' na presença de espécies. ${ }^{83}$

Nesse sentido, a 'perda nula' é outra dificuldade que se apresenta. Os requisitos para demonstrar a ausência de 'perda líquida' de uma ação de compensação, geralmente, não estão bem definidos e as linhas de base pouco especificadas. As linhas de base são normalmente fixas às condições da biodiversidade até o momento do projeto de desenvolvimento tomar seu lugar. ${ }^{84}$ Supondo que um ambiente em um avançado estágio de degradação (ou vulnerável em função de seu estágio de suscetibilidade) venha a ser compensado em função de sua supressão. Se o projeto de compensação agregar as tendências de degradação do ambiente (já degradado e suscetível), qualquer ação que mantenha o estágio de degradação atual em qualquer outro lugar escolhido para a compensação poderá representar uma 'perda nula', se a linha de base for fixa. Pode-se até abrir margens para compensar um dano à biodiversidade por outro lugar que esteja também em estágio degradado, não oferecendo adicionalidade em termos de conservação.

Além disso, a linha de base fixa tende a não considerar as interações na paisagem. A ecologia da paisagem, sobretudo em ambientes em que as pressões antropogênicas se mostram acentuadas, tem recebido bastante relevância. É fundamental que um esquema de BDO atenda a critérios específicos de acordo com a escala. Não obstante, há sempre a possibilidade de 'vazamentos' (leakage) para fora da área de atuação

81 Maron et al., 2012; Hannis e Sullivan, 2012.

82 Pawliczek e Sullivan, 2012.

83 Como afirmam Hannis e Sullivan, (2012, p. 9): "The actual efficacy of these schemes seems hard to establish with impartiality or certainty".

84 Bull et al., 2013. 
do projeto de compensação, especialmente quando um projeto desconsidera as interações através da paisagem. ${ }^{85}$ As recomendações de Bull et al. (2013) passam por mensurar a 'perda nula' baseando-se em uma linha de base dinâmica, de modo a incorporar as trocas e tendências, especificar a escala (nível do projeto ou paisagem) e considerar aplicar taxas de descontos de modo que as trocas entre as perdas no presente e os ganhos no futuro possam se equivaler.

As taxas de desconto apresentam dificuldades à parte. A taxa de desconto $^{86}$ é um processo de escolha em que se investe na formação de capital no futuro em detrimento do consumo presente, sendo um processo intrinsecamente associado à produtividade de capital. ${ }^{87} \mathrm{~A}$ taxa de desconto direcionada para o contexto da compensação de biodiversidade implica considerar a biodiversidade enquanto um capital ('capital natural', mas ainda sim, um capital). Os projetos de compensação, a princípio, violam qualquer princípio de insubstituibilidade, na medida em que visam compensar um dano, muitas vezes 'off-site'. O 'capital natural' que se degrada no presente para sua respectiva compensação no futuro desconsidera as relações intrínsecas com outros elementos naturais intangíveis que não foram (monetariamente) quantificados. ${ }^{88} 89$ Para além disso, uma queda na taxa de desconto pode ter dois efeitos distintos $^{90}$ : um efeito de conservação no presente visando a preservação de uma área para o futuro (contrário aos ideais da compensação que visam a degradação presente para uma compensação futura); e um efeito de des-

$\overline{85 \quad \text { Bull et al., } 2013 .}$

86 A taxa de desconto é considerada um juro alternativo em que compara medidas de valor através do tempo. Ao lado disso, o desconto é um referencial da produtividade de capital, em que se investe na formação de capital no futuro em detrimento do consumo presente.

87 Mata e Cavalcanti, 2002.

88 Lumley, 1997.

89 O capital natural pode, segundo a economia ambiental ser compensado por outro capital, desde que a produtividade de capital não entre em declínio. As relações entre a biodiversidade degradada no presente com outros elementos naturais circundantes são desconsideradas. A eficiência econômica, guiada pelo desconto dos fluxos de custos e benefícios, poderá levar à exaustão da biodiversidade no presente. E mesmo ao lado da economia ecológica, a racionalidade do desconto não pressupõe um retorno do mundo natural, mas pressupõe que não haja declínio de capital natural.

90 Mata e Cavalcanti, 2002. 
investimento, na medida em que uma taxa baixa de desconto diminui os custos de exploração, encorajando o processo de supressão ambiental. Se a taxa de desconto é um processo de escolha intertemporal, implica decidir o que é o melhor para as partes. Para o mundo corporativo, o melhor é o ganho financeiro, a curto prazo, na medida em que objetivo final é o lucro. ${ }^{91}$ Nesse sentido, o desconto não favorece um cenário em que não há 'perdas líquidas', ao contrário favorece a degradação presente; a criação de créditos alheios às complexidades ecológicas; um contexto de incerteza no momento de fazer com que estes créditos sejam efetivamente transformados em áreas de conservação.

Há ainda uma enorme incerteza associada à questão da «perda nula'. Não está assegurado que tais mecanismos de compensação conseguirão criar uma rede em que há 'perda nula' de biodiversidade (quanto mais um 'impacto positivo'). ${ }^{92}$ Maron et al. (2012) argumentam que o domínio no âmbito da restauração em que se possa atingir a 'perda nula' de biodiversidade é muito reduzido e concluem que não há um suporte de evidências que satisfaça as expectativas depositadas sobre a restauração por parte dos proponentes dos esquemas de compensação. ${ }^{93} \mathrm{~A}$ habilidade da restauração ecológica de corresponder à biodiversidade que sofreu a perda (ou o dano) é limitada pela dificuldade de se mensurar os valores que se perderam e se ganharam, considerando ainda as incertezas e o tempo que se leva para as medidas de restauração terem efeitos. ${ }^{94}$

Os mecanismos de compensação de biodiversidade depositam grande esperança nas estratégias de restauração para se gerar os créditos de biodiversidade (para se compensar a perda ou transacionar a biodiversidade para futuros empreendimentos). ${ }^{95}$ É fundamental reparar que isso fez com que as políticas de compensação se tornassem um dos

\footnotetext{
$91 \quad$ Lumley, 1997.

92 Waeber, 2013.

93 Maron et al., 2012.

94 Maron et al. (2012) sugerem três categorias fundamentais em que a restauração ecológica não pode suportar a compensação da biodiversidade: i) pouca qualidade na mensurabilidade dos valores a ser compensados; incerteza nos resultados da restauração; desfasamento temporal, isto é, depois de suplantados os desafios da mensuração e da incerteza, quanto tempo depois se verão resultados?
}

95 Maron et al., 2012. 
maiores drivers das atividades de restauração ecológica. ${ }^{96}$ Mas é importante salientar que a ciência da restauração ecológica está ainda em fase experimental, sem um corpo sólido de evidências, sendo que a dinâmica dos sistemas naturais leva a reconhecer que é pouco provável e fiável que a restauração atingirá os padrões de integridade anteriores à degradação. ${ }^{97}$

Os pagamentos poderiam ainda gerar um efeito contraproducente, segundo a linguagem econômica, um efeito perverso: condicionar as ações pró-ambientais aos pagamentos monetários. É o que se chama de 'crowding out' 98 das motivações de cunho moral, estético ou de cuidado relativamente à conservação, isto é, comprometendo tais motivações intrínsecas, condicionando-as aos pagamentos. ${ }^{99}$

Um estudo de Narloch et al. (2012) aponta que em comunidades nas quais há um maior comprometimento institucional com a conservação, os instrumentos baseados em mercado poderão fomentar uma situação de crowd-out, mas poderão desencadear uma situação de crowd-in onde os laços institucionais estão fragilizados e poucas são as motivações para a conservação. No entanto Rode et al. (2015), apontam que há muito mais evidências de crowd-out. Ao considerar a complexidade de se avaliar em quais condições ocorre crowd-out, um projeto deve avaliar as motivações, suas estruturas metodológicas e o contexto socioecológico no local em que se visa a implementação do projeto.

Nessa medida, seria uma tarefa sem sentido produzir um ganho geral para a natureza a partir de sua fragmentação. Portanto, não seria concebível uma situação de 'no net loss' tampouco de 'net gain' de biodiversidade gerada a partir da rutura das dinâmicas e conexões entre indivíduos, populações e lugares, legitimada pela estratégia de BDO.100

96 ten Kate et al., 2004; Maron et al., 2012.

97 Maron et al., 2012.

98 A hipótese do 'crowding-out' deriva do argumento de Richard Titmuss de que os doadores de sangue não são motivados pelo dinheiro, mas por questões morais, sendo que a compensação monetária para fazê-lo criou uma situação de 'crowd out' na oferta de sangue, ou seja, as doações de sangue diminuíram, ao invés de aumentar, como era esperado, Kosoy e Corbera, 2010.

99 Kosoy e Corbera, 2010; Hannis e Sullivan, 2012; Gordon et al., 2015.

100 Hannis e Sullivan, 2012. 
O quarto princípio discutido por Hannis e Sullivan (2012) são as 'métricas ecossistémicas para construir comensurabilidade'. A métrica é um dos principais sobressaltos acerca dos esquemas de BDO. ${ }^{101}$ Como já referido, não existem métricas que possam representar a complexidade da biodiversidade e suas intrínsecas relações ecológicas, tampouco, o valor inestimável que a cerca. O próprio conceito de biodiversidade sofre com ambiguidades conceituais e controvérsias no seu uso. No entanto, as compensações depositam sua confiança na quantificação acurada das perdas e ganhos da biodiversidade, requerendo, por isso, métricas bastante fiáveis. As métricas compostas consideram mais de um fator (como as condições das vegetações e seu contexto espacial), sendo mais apropriadas; enquanto as métricas singulares como 'área de habitat' vêm perdendo relevância. ${ }^{102}$ Ainda sim, as métricas compostas não são capazes de captar a diversidade genética, tampouco, o caráter evolutivo das espécies entre outras características multidimensionais da biodiversidade. Entretanto, as métricas compostas, em função de suas complexidades, podem acabar por não cumprir seu papel, arriscando-se ao reducionismo das dimensões em área do habitat, muito mais fáceis de mensurar.

A escolha das métricas influencia na maneira como os ganhos e perdas serão contabilizados e também em como será definida a perda nula. E, além disso, haverá implicações para o cálculo de descontos (ratios) em contextos de incertezas e na escolha do local para as compensações. ${ }^{103}$ As sugestões de Bull et al. (2013) para uma operacionalização mais adequada dos BDO passam pela utilização das métricas compostas, bem como utilização de outros fatores agregados, como as funções ecossistêmicas. Mas ao lado disso, a utilização das métricas agregadas poderão dificultar a avaliação das equivalências e das ações de restauração. ${ }^{104}$

Mesmo diante de grandes esforços científicos, como averiguar a importância e equivalência ecológica entre áreas naturais? Os créditos de

101 Bull et al., 2013; Gonçalves et al., 2015.

102 Bull et al., 2013.

103 Gonçalves et al., 2015.

104 Gonçalves et al., 2015 
biodiversidade podem ser gerados antes ou depois, em qualquer localidade, para que a biodiversidade possa ser compensada em qualquer momento e para qualquer dano. Existe uma dificuldade inerente em atestar a equivalência entre 'as biodiversidades', segundo O'Neill (1997), a incomensurabilidade entre os elementos naturais. Mesmo quando as transações são feitas entre indicadores análogos, por exemplo, entre habitats pertencentes a um mesmo contexto ecológico, a equivalência não pode ser assegurada. Desta forma, traçar equivalências ecológicas entre os elementos naturais que diferem em tipo, tempo, lugar e contexto ecológico se torna uma tarefa pouco provável de ser cumprida.

Bull et al. (2013) afirmam que a hipótese de uma troca de um habitat com pouca significância ecológica (local afetado pelo desenvolvimento) por um habitat com elevado valor ecológico (sítio onde se deu a compensação) até pode ser um poderoso argumento, sobretudo para as trocas off-site. Contudo, aquele habitat, mesmo diante de pouco valor ecológico, pode ter um valor funcional sistêmico que poderá nunca ser restabelecido. Como se determina essa relevância ecológica? E mais, mesmo sem valor ecológico relevante, este habitat não teria o 'direito' de florescer, não carrega em si um valor próprio, independente de sua relevância para os seres humanos e também de sua relevância ecológica?

Ao passo em que se incentiva a transação de créditos de conservação através de localidades distintas, requer-se uma medida única numérica para comensurar as áreas conservadas entre si: o preço.

As críticas que recaem sobre os esquemas de compensação da perda de biodiversidade são multivariadas e multissetoriais, e não se limitam aos termos da restauração ecológica. Não obstante, os aspetos relacionados com o desenho dos mecanismos de compensação, da governança de como tais créditos serão assegurados no campo, entre outras dificuldades como a própria ubiquidade ética também trazem implicações ao se considerar tais instrumentos para a conservação. ${ }^{105}$

Isso nos leva ao quinto princípio: 'habilitação política e a plataforma de governança'. Por mais que se declare que a razão principal dos 'bancos de conservação' (que possibilitam a criação de créditos para os biodiver-

105 Maron et al., 2012; Maron et al., 2016 
sity offsets) seja a manutenção da saúde ambiental através de transações financeiras entre mercados privatizados, é inegável que o setor público, bem como as regulações governamentais, representam um papel indispensável para sua operacionalização. ${ }^{106}$ Assim, os Governos são encorajados a legislar sobre a conservação baseada na transação de ativos ambientais (como habitats, espécies, florestas), situação emergente, por exemplo, no Brasil, tendência bem notada pela flexibilização do Novo Código Florestal Brasileiro. ${ }^{107}$ Contudo, o papel governamental nisso tudo não se limita à regulação, mas pode expandir-se utilizando-se de recursos para tornar o investimento privado atrativo através de subsídios e isenção de impostos entre outras medidas para reduzir os riscos do investimento privado. ${ }^{108}$ Aliás a falta do envolvimento público, nestes termos comprometeu todo o funcionamento dos mecanismos de BDO no Reino Unido. ${ }^{109}$

É importante ressaltar que os instrumentos de mercado precisam de uma regulação e de todo um sistema (fora do mercado) que facilite, ou mesmo que os tornem possíveis. Naturalmente, um mercado não é gerido apenas pelos preços ${ }^{110}$, mas por todos seus mecanismos e ferramentas associadas e, especialmente, pela ideologia que o constitui. Nesse sentido, interessa saber que, por todo o globo, o arranjo institucional está sendo redesenhado para que tais instrumentos baseados em mercado (sejam eles genuínos ou não) possam ser favorecidos. Pois mesmo quando tais instrumentos não são um mercado puro, os arranjos institucionais induzem as decisões a serem tomadas como se estivessem em um esquema de mercado.

A legislação americana, por exemplo, suporta o estabelecimento de bancos de espécie para financiar a conservação das espécies através da transação de créditos. Desta forma, os proprietários de terras podem compensar (offset) suas atividades nocivas ao ambiente através da compra de créditos de espécies, ou ainda, poderão eles buscar uma habil-

106 Hannis, e Sullivan, 2012; Santos et al., 2015.

107 Packer, 2015.

108 Hannis e Sullivan, 2012.

109 Lockhart, 2016.

110 Lapeyre et al., 2015. 
itação ao Fish and Wildlife Service (FWS), instituição responsável pelos bancos de créditos de áreas húmidas nos EUA, para que possam eles próprios compensar seus próprios danos em suas próprias propriedades. ${ }^{11}$ Medidas como essa permitem que se convertam áreas de conservação, legalmente exigidas, em produtos ambientais. Ao 'produzir' suas próprias compensações, os proprietários que provocaram o dano poderão ainda lucrar colocando os 'créditos' restantes no mercado.

Essa mesma lógica se aplica no caso do Brasil. Por exemplo, as Reservas Legais ${ }^{112}$, áreas de conservação obrigatória nas propriedades privadas rurais, com função socioambiental bem esclarecida, são hoje objeto de transação. ${ }^{113}$ Cria-se com isso uma autorização para se desmatar e compensar em áreas de terceiros, situação legitimada legalmente. Além de conceder, tanto ao setor público quanto ao privado, o 'direito' de destruir, desde que se compre 'créditos' de um 'banco de conservação' para compensar. ${ }^{114}$

Os BDO na União Européia também seguem em andamento em países como Alemanha, Finlândia, Suécia, França, e como já referido, no Reino Unido, a seguir as premissas da hierarquia da mitigação e existem estudos de viabilidade para países como Portugal, Espanha, Polónia, República Checa, Grécia, Lituânia, Holanda, Itália, Bulgária. ${ }^{115}$ Segundo estes estudos, tem havido crescimento significativo de florestas e bosques (arbustivos) e de áreas húmidas em Portugal, o que torna o país atrativo para aoperacionalização dos $\mathrm{BDO}^{116}$, especialmente de forma transfronteiriça através da União Européia.

111 Hannis, e Sullivan, 2012.

112 Reserva Legal é uma área localizada no interior de uma propriedade ou posse rural, delimitada nos termos do art. 12, com a função de assegurar o uso econômico de modo sustentável dos recursos naturais do imóvel rural, auxiliar a conservação e a reabilitação dos processos ecológicos e promover a conservação da biodiversidade, bem como o abrigo e a proteção de fauna silvestre e da flora nativa (c.f. http://www.planalto.gov.br/ccivil_03/_ato2011-2014/2012/lei/I12651.htm).

113 Packer, 2015.

114 Hannis e Sullivan, 2012.

115 eftec e IEEP, 2010; ICF GHK e Bio Intelligence Service, 2013.

116 ICF GHK e Bio Intelligence Service, 2013. 
Obviamente, esse tipo de conservação será benéfico para os empreendedores, na medida em que poderão prosseguir com seus empreendimentos sem muitas restrições de cunho ambiental. Muitas vezes, será razão de lucro para aqueles que criarão seus próprios créditos de conservação. $E$, claramente, não será benéfico para a biodiversidade nas localidades em que o desenvolvimento se dá de forma mais agressiva e intensa, considerando que a biodiversidade poderá ser compensada em qualquer outra localidade. A compensação pela biodiversidade será boa também para os proprietários de habitats inscritos nos bancos, incluindo proprietários privados, ou mesmo ONGs que poderão também converter os ganhos neste mercado para aprimorar e aumentar as áreas de conservação e restauração. Neste último caso, até poderia ser benéfico para a biodiversidade local, dependendo das circunstâncias nas quais o processo se estabelece. No entanto, na medida em que tais mecanismos de mercado para a conservação se tornam cada vez mais populares, acabam por legitimar a redução dos fundos para outros tipos de conservação ${ }^{117}$, como a criação de áreas protegidas.

Maron et al., (2016) argumentam que para que os BDO demonstrem algum benefício, devem ser aplicados com precaução, pois existem muito riscos associados, que devem ser minimizados, e diante de sua expansão acrítica, nem as melhores políticas seriam capazes de lidar com seus efeitos.

As atividades de desenvolvimento que geram os danos que os mecanismos de BDO pretendem compensar continuarão a causar danos ao ambiente natural. O que mudou com o passar do tempo é a estratégia de marketing utilizada pelas indústrias (empresas) responsáveis por esses danos: passaram a utilizar o mesmo discurso e as mesmas narrativas das organizações conservacionistas. ${ }^{118}$ Mas se tais mecanismos passam a ser aceitos sem olhar crítico e avaliação mais atenta, os desafios serão apenas uma questão de detalhes e de implementação ${ }^{119}$ para ditar os moldes da conservação da biodiversidade e, em última instância, nossa relação com a natureza.

117 Hannis e Sullivan, 2012.

118 Waeber, 2013.

119 Hannis e Sullivan, 2012. 
Neste processo, decompõe-se a natureza, em toda a sua diversidade, relacionalidade, interconectividade, não-linearidade e complexidade em unidades monetariamente mensuráveis. ${ }^{120} \mathrm{~A}$ representação numérica da complexidade da natureza visa a equivalência e a comensurabilidade entre os diferentes elementos naturais, tempo, localidade e entre os diferentes tipos de capital. ${ }^{121}$ Essa estratégia é o que conduz a novos instrumentos de conservação baseados em mecanismos de offsetting. ${ }^{122}$

\section{Considerações Finais: (des)significando a Conservação?}

A conservação da biodiversidade hoje está voltada para um lado objetivo-econômico, cada vez mais distante de questionamento. Nessa perspetiva, a natureza é marginal aos interesses que parecem mais evidentes: a eficiência econômica e, mais obscuramente, o lucro. Naturalmente, os objetivos da conservação devem ser defendidos de forma bastante objetiva e, por vezes, mesmo num sentido utilitário, mas as bases para as tomadas de decisões devem passar por uma reflexão ética fundamental. Um processo de decisão passa igualmente por um processo de julgamento de valores, no qual os interesses e as considerações são legitimadas e, certamente, uma questão de legitimação deve ser alvo de uma avaliação crítica. ${ }^{123}$

Alguns dos proponentes dos instrumentos económicos para a conservação argumentam que essa avaliação crítica tende a ser uma tentativa de frear o progresso de algo que tem grande potencial para o sucesso. ${ }^{124}$ No entanto, o espaço que tais instrumentos vêm a ocupar na conservação se deu de forma quase inquestionável e seu 'potencial para o sucesso' se tornou uma verdade quase absoluta ${ }^{125}$, ainda que não tenha sido, de facto, um sucesso. ${ }^{126}$ Esse espaço deve ser questionado, não como uma reação a novas estratégias para a conservação, obviamente, mas de forma a garantir que os interesses da natureza e de sua conser-

120 Sullivan, 2014.

121 Sullivan, 2014.

122 Robertson, 2006; Sullivan, 2013; Sullivan, 2014.

123 Holland e Rawles, 1994.

124 Wunder, 2013.

125 Sullivan, 2014.

126 Lockhart, 2016. 
vação sejam assegurados, especialmente em um contexto de incertezas e mudanças globais (sociais e ambientais) sem precedentes, como as alterações climáticas, que nos demandam uma contínua reflexão crítica. ${ }^{127}$

Não é conclusivo, mesmo pouco provável, que a estratégia da compensação possa constituir uma ajuda fundamental para a resiliência ecológica, ou para se atingir o que se chama de 'sem perda líquida de biodiversidade'. Contudo, mais fundamental ainda, não é se tal abordagem funciona ou não, mas o quê a estratégia da compensação da biodiversidade nos diz a respeito da relação humana com o mundo não humano. A compensação não pode, no âmbito de sua definição, fomentar qualquer relação específica entre uma comunidade e seus indivíduos, sua paisagem e com o mundo natural não-humano que a rodeia. Ao contrário, a ideia preservada em uma política baseada na compensação entre as mais diversas formas de vida e ecossistemas, simplifica e retrocede a perceção humana sobre o mundo natural, fazendo com que a Natureza seja entendida como fragmentos iguais uns aos outros. ${ }^{128}$ Como ainda Hannis e Sullivan 129: "This is clearly not serving the broader cause of conservation, and risks undermining the work of the many organisations, both public and private, who strive to (re)build caring and attentive connections between people and their local environments".

É uma perspetiva que permite a criação de espaços humanos dos quais a natureza sequer faz parte, e o isolamento da natureza na esfera humana como se esta última sequer fizesse parte. É importante perceber que esta é uma 〈polarização〉 que ofusca a necessidade de se reforçar formas de viver nas quais se estimula uma integração entre o habitat natural e o mundo humano. ${ }^{130}$

Pode o projeto de compensar por uma 'biodiversidade' desaparecida ser considerado conservação? Toda a vida 'real' que ali existia já não existe mais, desvaneceu-se com a história do lugar, e perde-se assim o sentido da conservação. De fato, sob essa perspetiva, não houve sequer conservação, uma vez que o que restou foi um crédito de biodiversidade,

127 Holland e Rawles, 1994; Santos, 2011.

128 Hannis e Sullivan, 2012.

129 Hannis e Sullivan, 2012, 15.

130 Hannis, 2011; Hannis e Sullivan, 2012; Turnhout et al., 2013; 
uma abstração, uma representação numérica da biodiversidade realocada em outro lugar qualquer. ${ }^{131} \mathrm{O}$ que se conservou, portanto, foi uma parcela transformada e descontextualizada da natureza, uma parcela desterritorializada. ${ }^{132}$

Na medida em que a vida humana está condicionada à saúde do sistema ecológico, o ônus da prova é uma questão essencial ${ }^{133}$ e deve repousar sobre essas novas estratégias de conservação desenhadas sob moldes desenvolvimentistas. ${ }^{134}$ Se, como sugerem Holland e Rawles (1996), a conservação se trata de negociar a transição do passado para o futuro de modo a assegurar um máximo de significância tanto para a vida humana quanto para a vida não-humana, é importante que haja uma atenção especial nessas novas tendências para a conservação, para que não se perca o significado e o sentido de se conservar.

\section{Referências}

Business and Biodiversity Offsets Programme (BBOP), 2009, Business, Biodiversity Offsets and BBOP: An Overview. Washington, DC: Forest Trends.

Business and Biodiversity Offsets Programme (BBOP), 2012, Biodiversity Offsets: Principles, Criteria and Indicators. Washington, DC: Forest Trends.

Beckert, C., and Varandas, M.J., 2004, Éticas e políticas ambientais. Lisboa, Centro de Filosofia da Universidade de Lisboa.

Böhm, S., \& Dabhi, S., 2009, Upsetting the Offset: The Political Economy of Carbon Markets. London: May Fly Books.

Bull, J.W., Suttle, K.B., Singh, N.J., Milner-Gulland, E.J., and Gordon, A., 2013, Biodiversity offsets in theory and practice. Oryx, 47(3), 369-380.

131 Hannis e Sullivan, 2012.

132 Deleuze e Guattari, 1989.

133 Rosa e Marques da Silva, 2005; Marques da Silva, 2012.

134 Holland e Rawles, 1994; Rolston, 1995. 
Büscher, B., Sullivan, S., Neves, K., Igoe, J., and Brockington, D., 2012, Towards a Synthesized Critique of Neoliberal Biodiversity Conservation, Capitalism Nature Socialism, 23(2), 4-30.

Callicott, J.B., 1984, Nonanthropocentric Value Theory and Environmental Ethics. American Philosophical Quarterly, 12, 299-309.

Callicott, J.B., 1986, On the Intrinsic Value of Nonhuman Species. In: B. Norton (Ed.), The Preservation of Species, Princeton, Princeton University Press.

Castree, N., 2003, Commodifying what nature? Prog Hum Geogr, 23, 273-297.

Crutzen, P.J., and Stoermer, E.F., 2000. The Anthropocene. Global Change Newsletter, 41(41), 17-18.

Dean, W., 1996. A ferro e fogo: a história e a devastação da Mata Atlântica brasileira, São Paulo, Companhia das Letras.

Deleuze, G., \& Guattari, F., 1987, A thousand plateaus: capitalism and schizophrenia, Minneapolis, University of Minnesota Press.

Economics for the Environment Consultancy (eftec) and Institute for European Environmental Policy (IEEP), 2010, The use of market-based instruments for biodiversity protection - The case of habitat banking Technical Report. http://ec.europa.eu/environment/enveco/index.htm. Accessed 15 Dezembro 2018.

Foster, J., 1997, Introduction: Environmental value and the scope of economics. In: J. Foster (Ed.), Valuing nature? Ethics, economics and the environment, London, Routledge, pp. 1-20.

Froger, G., Ménard, S., \& Méral, P., 2014, Towards a comparative and critical analysis of biodiversity banks, Ecosystem Services, 1-10.

Gibbons, P., and Lindenmayer, D.B., 2007, Offsets for land clearing: No net loss or the tail wagging the dog? Ecological Management and Restoration, 8(1), 26-31.

Gillenwater, M., 2012, What is Additionality? Part 1: A long stand problem. Discussion Paper 001. Greenhouse Gas Management Institute.

Gonçalves, B., Marques, A,, Soares, A.M.V.D.M., Pereira, H.M., 2015, Biodiversity offsets: From current challenges to harmonized metrics. Current Opinion in Environmental Sustainability 14, 61-67. 
Gordon, A., Bull, J.W., Wilcox, C. and Maron, M., 2015, Perverse incentives risk undermining biodiversity offset policies, Journal of Applied Ecology, 52, 532-537.

Gómez-Baggethun, E., de Groot, R., Lomas, P.L., and Montes, C., 2010, The history of ecosystem services in economic theory and practice: From early notions to markets and payment schemes. Ecological Economics, 69(6), 1209-1218.

Hannis, M., and Sullivan, S., 2012, Offsetting Nature? Habitat Banking and Biodiversity Offsets in the English Land Use Planning System. Techinical Report. Green House, Weymouth, UK.

Holland, A., and Rawles, K., 1994, The Ethics of Conservation: Report prepared for, and submited to Countryside Council for Wales. Lancaster.

ICF GHK e Bio Intelligence Service, 2013, Exploring potencial demand for and supply of habitat banking in the EU and appropriate designs elements for a habitat banking scheme. http://ec.europa.eu/environment/enveco/taxation/pdf/Habitat_banking_Report.pdf Accessed 5 Janeiro 2019.

Ives C. D., Bekessy S. A., 2015, The ethics of offsetting nature. Frontiers in Ecology and Environment, 13, 568-573.

.Kosoy, N., and Corbera, E., 2010, Payments for ecosystem services as commodity fetishism. Ecological Economics, 69(6), 1228-1236.

Lapeyre, R., Froger, G., and Hrabanski, M., 2015, Biodiversity offsets as market-based instruments for ecosystem services? From discourses to practices. Ecosystem Services, 1-9.

Larrère, C., and Larrère, R., 1997, Do Bom Uso da Natureza. Lisboa, Instituto Piaget.

Lockhart, A., 2016. Unnatural Markets: The politics of biodiversity offsetting and failed environmental market-making in England, PhD thesis, University of Sheffield.

Lumley, S., 1997, The environmental and ethics of discounting: an empirical analysis, Ecological Economics 20, 71-82.

Madsen, B., Carroll, N., Kandy, D., and Bennett, G., 2011 , State of Biodiversity Markets Report: Offset and Compensation Programs Worldwide. Washington, DC, Forest Trends. 
Maron, M., Hobbs, R.J., Moilanen, A., Matthews, J.W., Christie, K., Gardner, T., McAlpine, C. a., 2012, Faustian bargains? Restoration realities in the context of biodiversity offset policies. Biological Conservation, 155, 141-148.

Maron M., Gordon A., Mackey B.G., Possingham H.P., Watson J., 2015, Stop misuse of biodiversity offsets. Nature 523: 401-403.

Maron, M., Ives, C., Kujala, H., Bull, J.W., Maseky, F.J.F., Bekessy, S. et al., 2016, Taming a wicked problem: resolving controversies in biodiversity offsetting. BioScience, 66, 489-498.

Marques, J., 2012, Do Senciocentrismo ao Holismo Ético - perspectivas sobre o valor da bioesfera. In: A. Barbosa, F. Martins Vale and P. Costa (eds) Gravitações Bioéticas, Lisboa: Centro de Bioética da Faculdade de Medicina da Universidade de Lisboa, pp. 123-145.

Mata, H.T.C., and Cavalcanti, J.E.A., 2002. A Ética Ambiental e o Desenvolvimento Sustentável, Revista de Economia Política, 22(85), 170-185.

McCauley, D. J., 2006, Selling out on nature. Nature, 443(7107), 27-8. https://doi.org/10.1038/443027a.

Midgley, M., 2004, The Myths We Live By. London: Routledge.

Miller, T.R., Minteer, B. a., \& Malan, L.C., 2011 , The new conservation debate: The view from practical ethics. Biological Conservation, 144(3), 948-957.

Minteer, B.A., and Miller, T.R., 2011, The New Conservation Debate: Ethical foundations, strategic trade-offs, and policy opportunities. Biological Conservation, 144(3), 945-947.

Moreno-Mateos D., Maris V., Bechet A., Curran M., 2015, The true loss caused by biodiversity offsets. Biological Conservation 192, 552-559.

Morris, R.K.A., Alonso, I., Jefferson, R.G., and Kirby, K.J., 2006, The creation of compensatory habitat: Can it secure sustainable development? Journal for Nature Conservation, 14(2), 106-116.

Narloch, U., Pascual, U. and Drucker, A.G., 2012, Collective action dynamics under external rewards: experimental insights from Andean farming communities, World Development, 40(10), 2096-2107.

Norgaard, R.B., 2010, Ecosystem services: From eye-opening metaphor to complexity blinder. Ecological Economics, 69(6), 1219-1227. 
Norton, B.G., 2000, Biodiversity and environmental values: In search of a universal earth ethic. Biodiversity and Conservation, 9(8), 1029-1044.

O'Neill, J., 1997, Value Pluralism, Incommensurability and Institutions. In: J. Foster (Ed.), Valuing nature? Ethics, economics and the environment, pp. 75-88. London, Routledge.

O’Neill, J., 2005, Meta-Ética. In: D. Jamieson (Ed.), Manual de Filosofia do Ambient, Lisboa, Instituto Piaget, pp. 173-186.

O'Neill, J., Holland, A., and Light, A., 2008, Environmental values. New York: Routledge.

Packer, L., 2015, Novo código florestal e pagamentos por serviços ambientais: regime proprietário sobre os bens comuns, Curitiba, Juruá.

Pawliczek, J., and Sullivan, S., 2011 , Conservation and concealment in SpeciesBanking.com, USA: an analysis of neoliberal performance in the species offsetting industry. Environmental Conservation, 38(4), 435-444.

Pievani, T., 2014, The sixth mass extinction: Anthropocene and the human impact on biodiversity. Anthropocene - Natural and Man-made Alterations of the Earth, 25, (1), 85-93.

Rio Tinto QMM, 2008, Biodiversity Offsets Strategy-QMM Rio Tinto Madagascar: Draft working paper and issues for discussion.

Robertson, M.M., 2006, The nature that capital can see: Science, state, and market in the commodifi cation of ecosystem services, Society and Space, 24(3), 367-387.

Rode, J., Gomez-Baggethun, E., Krause, M., 2015, Motivation crowding by economic payments in conservation policy: a review of the empirical evidence, Ecological. Economics. 117, 270-282.

Rolston III, H., 1989, Philosophy Gone Wild (Second Edition). New York, Prometheus Books.

Rolston III, H., 1994, Value in Nature and the Nature of Value. In: R. Attfield and A. Belsey (Eds.), Philosophy and the Natural Environment, Cambridge, Cambridge University, pp. 13-30.

Rolston III, H., 1995, Global Environmental Ethics: A Valuable Earth. In: R.L. Knight and S. F. Bates (Eds.), A New Century for Natural Resource Management, Washington, Island Press, pp. 349-366. 
Rosa, H.D., and Marques da Silva, J., 2005, From environmental ethics to nature conservation policy: Natura 2000 and the burden of proof. Journal of Agricultural and Environmental Ethics, 18(2), 107-130.

Sandbrook, C., Scales, I.R., Vira, B., and Adams, W.M., 2011, Value plurality among conservation professionals. Conservation Biology, 25(2), 285-294.

Santos, R., Schroeter-Schlaack, C., Antunes, P., Ring, I., Clemente, P., 2015, Reviewing the role of habitat banking and tradable development rights in the conservation policy mix. Environmental Conservation 42(4), $1-12$.

Santos, F.D., 2011 , Humans on Earth: from origins to possible futures, London, Springer.

Schumacher, E.F., 1973, Small is Beautiful: Economics as if People mattered, London, Vintage.

Soulé, M.E., 1985, What is Conservation Biology? BioScience, 35 (1 1), 727-734.

Soulé M.E., 1996, The End of Evolution, IUCN World Conservation n. 1, p. 24.

Spash, C.L., 2009, The new environmental pragmatists, pluralism and sustainability. Environmental Values, 18(3), 253-256.

Spash, C.L., 2015, Bulldozing biodiversity: The economics of offsets and trading-in Nature (PDF) Biological Conservation 192, 541-551.

Sullivan, S., 2010, Ecosystem Service Commodities: A New Imperial Ecology? Implications for Animist Immanent Ecologies, with Deleuze and Guattari. New Formations, 69(1), 111-128.

Sullivan, S., 2013, Banking Nature? The Spectacular Financialisation of Environmental Conservation. Antipode, 45(1), 198-217.

Sullivan, S., 2014, The natural capital myth; or will accounting save the world? Preliminary thoughts on nature, finance and values.

The Economics of Ecosystems and Biodiversity (TEEB), 2010, The Economics of Ecosystems and Biodiversity: Mainstreaming the Economics of Nature: A Synthesis of the Approach, Conclusions and Recommendations of TEEB. http://www.teebweb.org/TEEBSynthesisReport/tabid/29410/ Default.aspx. Accessed 15 Dezembro 2018. 
ten Kate, K., Bishop, J., and Bayon, R., 2004, Biodiversity Offsets: Views, Experience, and the Business Case. London, Insight Investment.

Turnhout, E., Waterton, C., Neves, K., and Buizer, M., 2013, Rethinking biodiversity: from goods and services to "living with." Conservation Letters, 6(3), 154-161.

United Nations Framework Convention on Climate Change (UNFCCC), 1998, Kyoto Protocol to the United Nations Framework Convention on Climate Change. Japan.

Van Dyke, F., 2008, Conservation Biology: Foundations, concepts, applications (2nd ed.). Springer-Verlag.

Viveiros de Castro, E., 2013, Últimas notícias sobre a destruição do mundo. Conferência Curt Nimuendajú. São Paulo, Centro de Estudos Ameríndios da USP. http://www.socioambiental.org/pt-br/noticias-socioambientais/os-indios-sao-especialistas-em-fim-do-mundo-diz-o-antropologo-eduardo-viveiros-de-castro. Accessed 15 Dezembro 2018.

Waeber, P., 2013, Biodiversity offsetting en vogue in Madagascar? Madagascar Conservation \& Development, 7(3), 110-111.

White, W., 2008, The Advantages and Opportunities. In: N. Carroll, J. Fox, and R. Bayon (Eds.), Conservation and Biodiversity Banking: A Guide to Setting Up and Running Biodiversity Credit Trading Systems, London, Earthscan, pp. 33-42.

Wilson, E.O., 2002, The Future of Life. New York, Vintage Books.

Wunder, S., 2013, When payments for environmental services will work for conservation. Conservation Letters, 6(4), 230-237. 